\title{
La protección social en América Latina y la particularidad de la asistencia social ${ }^{1 /}$ Social protection in Latin America and the particularity of social assistance
}

Resumen: El articulo tiene como objetivo analizar la particularidad de la asistencia social, en el contexto de la protección social en América Latina y los desafíos por la ampliación de los derechos sociales, teniendo en cuenta la predominancia de políticas focalizadas, donde ocupan destaque los Programas de Transferencia Condicionada (PTC), en contraposición a las políticas universales, que cada vez más tiene su acceso restringido y mercantilizado. Destacan se las orientaciones de los organismos internacionales basadas en la individualización de las responsabilidades con el bienestar, por medio del incentivo al desarrollo de las capacidades humanas, del espíritu emprendedor, de la activación para el trabajo. De titular al derecho a responsable y deudor por la asistencia recibida, se va ampliando la moralización de los ciudadanos.

Palabras Clave: América Latina; asistencia social; transferencia condicionada; focalización; activación.

Abstract: The article aims at analyzing the particularity of social assistance, in the context of social protection in Latin America and the challenges for the expansion of social rights, taking into account

1 El contenido de esto artículo, tiene como base la exposición hecha en el X Congreso Nacional y VII Internacional de Trabajo Social. Tema Central "Trabajo Social Latinoamericano y del Caribe: Resistencias y propuestas frente a las desigualdades económicas, sociales y políticas". Realizado por el Colegio de Trabajadores Sociales de Costa Rica, en San José, 19 al 21 de julio del 2017 (Panel: Trabajo profesional en el campo de la justicia, la salud y la asistencia social).

2 Trabajadora Social (1982), Especialista (1985) y Maestra en Política Social (1997), Doctora en Sociología (2005). Docente del Departamento de Trabajo Social y del Programa de Postgrado en Política Social/UnB. Investigadora del Grupo de Estudios e Investigaciones en Seguridad Social y Trabajo (GESST). 
the predominance of focalized policies, where they occupy highlight programs conditional transfer (PTC), as opposed to universal policies, which increasingly have their access restricted and commodified, in line with the guidelines of international agencies based in the individualization of responsibilities welfare, through the incentive to the development of human capabilities, the entrepreneurial spirit of activation to work. Holder the right to responsible and debtor by the assistance received, it extended the moralization of the citizens.

Keywords: Latin America; social assistance; cash transfer; focus; activation.

$\mathrm{H}$ ablar de la protección social, en especial de la asistencia social en los tiempos actuales, o sea, en la fase contemporánea del capitalismo, marcado por el avance del conservadorismo, por el dominio del capital en todas las partes del Planeta, constituye un gran desafío. Impone comprendernos el sentido y los fundamentos que subsidian la formulación de políticas públicas. Para esto, buscamos algunos indicadores sociales que expresan la configuración de la realidad latinoamericana y las respuestas del Estado frente a las desigualdades económicas, sociales y políticas. Los indicadores evidencian el crecimiento del gasto social público, pero la predominancia de políticas focalizadas, en contraposición a las políticas universales, donde ganan especial protagonismo los Programas de Transferencia Condicionada, considerados por los organismos internacionales como una de las principales innovaciones en el campo de la política social latinoamericana en las últimas dos décadas. Verificase en el citado periodo, las orientaciones de que los programas sociales deben estar direccionados a los más necesitados, con el argumento del uso eficiente de los recursos financieros y de la inclusión social, haciendo con que los países de América Latina se utilicen de políticas focalizadas, con base en severas líneas de pobreza. Esto horizonte, impone la comprensión de esta contradictoria dinámica, entre la mercantilización de los servicios sociales y la monetización de la protección social, donde se distinguen los trabajadores entre aquellos que pueden adquirir la protección provista por el mercado y aquellos, más pobres, precarizados y los desempleados, que necesitan de la intervención del Estado. (GRANEMANN, 2007). 
La exposición del contenido está organizada de modo a localizarnos la configuración de la protección social en la región latinoamericana, a partir de las tendencias de las políticas sociales al largo de las últimas dos décadas, destacando orientaciones de organismos internacionales; los aspectos generales de los PTC en la región y las tendencias de activación para el trabajo en los mismos.

\section{La realidad latinoamericana en las últimas décadas}

\section{Protección social latinoamericana: evolución y modelos de desarrollo}

La historia de la protección social en la región es identificada a partir de la historia de sus modelos de desarrollo y sus respectivos paradigmas económicos y sociales. Cecchini y Martínez (2011) identifican esta evolución a partir de cuatro momentos: el primer, en el comienzo del siglo XIX, hasta la crisis de 1929, influenciado por el pensamiento liberal, dominado por el modelo exportador primario. Los indicadores sociales se caracterizan, por un lado, por la conformación de las sociedades nacionales y el sentido de pertenencia de los ciudadanos a su país y, por otro, la caridad, donde la atención a los problemas sociales es hecha por medio de ayuda a los más necesitados, por organizaciones sociales y la iglesia. A fines de los años 1920, empiezan a surgir los primeros sistemas de seguridad social inspirados en el modelo bismarckiano. El segundo momento, va de inicio de los años 1930, hasta finales de los 70, se caracteriza por el modelo substitutivo de importaciones, cuando los temas sociales se relacionaban con la justicia, el orden social y la seguridad social, destacándose la repercusión y papel de la sociedad organizada y los sindicatos. Las limitaciones de cobertura comprometen la perspectiva universalista de la política social, priorizándose la protección contributiva, a los trabajadores asalariados, en cuanto la protección no contributiva, restringida a los grupos más vulnerables. En esto período, la gestión de las políticas sociales, es marcada por la planificación central y el financiamiento, predominantemente, estatal, con escasa participación del sector privado.

El tercer momento, en fines de los años 70 y principios de los 80 , es marcado por la crisis de la deuda, el déficit fiscal, transformaciones del capitalismo industrial nacional en capitalismo globalizado, financiero y de servicios (FILGUEIRAS, 2013). Las recomendaciones del 
Consenso de Washington y la creencia de que el mercado es el mejor instrumento para asignar bienes y servicios, caracterizan el enfoque neoliberal de crecimiento hacia afuera. La austeridad fiscal, el ajuste estructural, los programas de estabilización económicos, con vistas a fomentar el crecimiento no llevó en consideración las desigualdades en la distribución de renta. Se en el momento anterior, el Estado era considerado el proveedor, en este, el Estado es subsidiario del mercado, que pasa a asumir papel central, no solo en el ámbito económico, pero también, en el social. La racionalización de los recursos, la eficiencia en la gestión constituye el argumento que conducirá las políticas sociales focalizadas en los más pobres. También, la descentralización asume relevancia, pues la gestión pasa a contar con la participación privada y de organizaciones no gubernamentales. Así, la protección social en el período asume característica dual, o sea, protección contributiva de los asalariados y, no contributiva, para amenizar la situación de la pobreza extrema, por medio de acceso a niveles mínimos de bienestar. A pesar de la institucionalidad democrática y algunas acciones concretas del Estado en el área económica y social, Latinoamérica presentó elevados niveles de desigualdad y pobreza, durante los años 80 y 90 .

El modelo de desarrollo desde mediados de los 90, fue basado en el "paradigma de la competitividad sistémica", donde se requiere incorporar progreso técnico al proceso productivo, con miras a elevar la productividad. La política social, como afirma Franco (1996), pasa a tener gran relevancia por su contribución a la formación de capital humano, ya que este es considerado indispensable a la competitividad de los países en el medio plazo.

Con la apuesta al capital humano, llega el siglo XXI, trayendo consigo, el "giro a la izquierda" de los gobiernos de la región y la incorporación de nuevas concepciones en el abordaje de la política social, como la garantía de derechos, ciudadanía plena, cohesión social, revelando preocupaciones con mejor distribución de los beneficios de desarrollo y con la disminución de la desigualdad. En la agenda, la tensión entre universalismo y focalización; en la administración de la política, la consideración de variables relativas a diferencias étnicas y culturales, de género, edad y lugar, además de las socioeconómicas. Igualmente, la valoración de los principios de efectividad y eficiencia, de la articulación público-privada, del papel regulador del Estado, pero 
no necesariamente proveedor y tampoco solamente subsidiario. La protección social pasa a convivir con debates y políticas que refleten la referida articulación público-privada favorecedoras del mercado: modelos de capitalización individual con solidaridad; la flexiseguridad laboral y el derecho a un ingreso mínimo. (CECHINNI; MARTÍNEZ, 2011).

En el contexto de las últimas décadas, América Latina, fue identificada como una región que ha ampliado la protección social, que "aproximadamente 40 millones de personas salieran de la pobreza para ingresar en la clase media” (MORENO, 2011, p. XIV), tras las amenazas por las turbulencias financieras internacionales provocadas por la crisis de 2008. Dados del BID (2016) y de la CEPAL ${ }^{3}$ (2016), demuestran la reducción en más de la mitad, del índice de pobreza extrema en la región, entre 1996 y 2014, de 29,9\% para 12,9\%.

En 2014, la pobreza ${ }^{4}$ en América Latina fue de 28,2\%, en cuanto el número de personas pobres alcanzó a 168 millones de personas, de las cuales 70 millones se encontraban en situación de indigencia. Hubo un crecimiento básicamente entre las personas pobres no indigente, cuyo número pasó de 166 millones en 2013 a 168 millones en 2014 (p. 18), un incremento alrededor de 2 millones de personas, entre 2013 y 2014. (CEPAL, 2016).

Sin duda, la reducción de los referidos niveles de pobreza e indigencia son significativos e importantes para la población del coyunto de los países de la región. Entretanto, persiste el elevado nivel de desigualdad que, en promedio, "por cada 100 unidades monetarias que percibió el $40 \%$ más pobre de la población, el 10\% más rico contó con 1.400 unidades monetarias", o sea, "el ingreso per cápita de las personas del decil más rico fue 14 veces superior en 2014 en comparación con el correspondiente a los cuatro primeros deciles". (CEPAL, 2016, p. 14).

3 CEPAL. Panorama Social da América Latina 2015. Documento Informativo, 2016.

4 La línea de pobreza utilizada es de US\$ 3,10 por día per cápita a la PPC de 2011, que es equivalente a la media de las líneas nacionales de pobreza extrema, o sea, el cuesto medio de una canasta básica en los países de la región. (CEPAL, 2016). 
La evaluación de CEPAL es que en el periodo posterior a la crisis financiera internacional (2008 y 2015), hubo una reducción de la desigualdad en la distribución de ingresos en la mayoría de los países, con el incremento de los ingresos del quintil inferior mayor que el observado en el quintil superior, desde los ingresos laborales (asalariados o trabajo independiente), las jubilaciones y las transferencias. Importante llamar a la atención para la desigualdad de ingresos desde la perspectiva de género, cuando un $44 \%$ de las mujeres de hogares del primer quintil, no cuentan con ingresos propios, en cuanto en el caso de los hombres, solamente un $23 \%$. Estos ingresos, en el caso de las mujeres, se componen el $16,8 \%$ de las transferencias y el $23,3 \%$ proveniente del trabajo independiente. La composición de los ingresos para los hombres es de $10 \%$ y $33,7 \%$ respectivamente. Sin embargo, "solamente una de cada dos mujeres en edad de trabajar tiene o busca un empleo, al mismo tiempo que las mujeres perciben en promedio únicamente el 83,9\% que reciben los hombres". (CEPAL, 2017, p. 9).

Otro elemento fundamental en el crecimiento de la desigualdad social es la estructura de la propiedad y de la riqueza en el mundo. Según dados de CEPAL (2017, p. 12), "0,7\% de los adultos (35 millones de personas) concentran casi la mitad (45\%) de los activos físicos y financieros del mundo; un total de solo 123.000 personas con un muy elevado patrimonio (que poseen más de 30 millones de dólares) acaparan el $9 \%$ de la riqueza mundial y 1.722 pertenecen al selecto grupo de los milmillonarios (billonarios)". También, a partir de las mediciones de desigualdad, en América Latina, basadas en datos tributarios, conforme CEPAL (2016 p. 27), se puede verificar la evolución de la participación del $1 \%$ más rico en ingreso total, entre 1992 y 2014, entre ellos, Argentina, Colombia, Uruguay y otros países y regiones. Colombia es el país con la mayor participación del $1 \%$ más rico en el ingreso total, que, en 2010, capturaba el 20,5\% del ingreso total del país. En Argentina y Uruguay, por su vez, los niveles de participación de los más ricos en el ingreso total eran superiores a los verificados en los países continentales de Europa Occidental y en el Asia Sudoriental. En Argentina en el año 2007, la participación del $1 \%$ más rico alcanzó el $16,8 \%$, en Uruguay fue de $14 \%$ en 2012 , y México, en 2012, fue de 21\%. Mientras tanto, entre los países desarrollados, el país con mayor participación de la población más rica en el ingreso total es Estados Unidos. 
En otras dimensiones se puede resaltar, cf. CEPAL (2016), logros alcanzados en la región cuanto a los niveles de escolaridad, veamos: en 2013, 92\% de la población de 15 a 19 años había concluido de la educación primaria; $58 \%$ en la educación secundaria; $80 \%$ de los jóvenes de 20 a 24 ańos del quintil más rico había concluido la secundaria, mientras que en el quintil más pobre esta tasa solo llegaba al 34\%. En la educación postsecundaria, entre 1997 y 2013, amplió la cobertura de la educación técnica o superior, incompleta o completa, pero su alcance fue de $46 \%$ en el quintil más rico y solamente el $4 \%$ en el quintil más pobre. En el mismo período, la ampliación de la escolaridad media para la población de 15 años o más en todos los quintiles.

En el área de acceso a vivienda y servicios sociales, CEPAL (2016) informa que entre 2002 y 2013, hubo una disminución del acceso insuficiente, pasando de un $22 \%$ al $14 \%$. No obstante, alrededor de 2013, el quintil más pobre tenía la tasa de acceso insuficiente a servicios básicos 4,5 veces la observada en el quintil más rico. Esta disparidad también se verifica en la incidencia de la habitación en viviendas con materiales precarios en $31 \%$ del decil de menores ingresos y al $26 \%$ en el segundo decil.

Frente a los indicadores destacados, importa verificar en qué medida las políticas utilizadas en la región, además de contribuyeren para su mejora, lo hacen también en la perspectiva de la concretización de derechos sociales y fortalecimiento de la ciudadanía. Se buscamos identificar las características de la protección social en la región, constatase que en las últimas décadas hubo retracción del papel del Estado en el social y la gestión centrada en la lógica de la eficiencia y disciplina fiscal, impactaran negativamente, en diferentes políticas sociales: En la área del trabajo, la desreglamentación y flexibilización contractual, generaran el fomento al trabajo por cuenta propia y al trabajo informal; en la área de la salud, la re mercantilización de la cobertura por medio de la gestión privada; en la área de los servicios sociales, la descentralización, e privatización de parte de los sistemas educativos y de salud; en el ámbito de las pensiones, América Latina fue pionera mundial en privatización e influenció otras regiones con reformas semejantes.

En reciente trabajo, Sojo (2017) analiza las tendencias de afiliación de los asalariados a los sistemas de salud y pensiones, donde se evidencia: el incremento de la inserción laboral y afiliación de las muje- 
res; las disparidades por quintiles, según niveles educativos; la heterogeneidad y diversidad dentro de los países sobre la cobertura de los asalariados. Según Sojo (2017, p. 65), en el periodo entre 2002 y 2013, los países con mayores coberturas de afiliación en pensiones son Argentina, Brasil, Chile, Costa Rica, la República Dominicana y Uruguay (zonas urbanas), cuyos niveles fluctúan entre un $68,5 \%$ y un $86,4 \%$. En salud, las mejores coberturas son de Colombia, México y la República Dominicana, que varían desde un $77,8 \%$ a un $99,1 \%$, que sin embargo tienen menores coberturas en pensiones.

Un otro grupo, compuesto por el Ecuador (zonas urbanas), El Salvador, el Perú presentan coberturas en salud que varían entre un $51 \%$ y un $67,9 \%$ de los asalariados. La cobertura en pensiones incluye también, Venezuela (República Bolivariana de), que decrece en el decenio analizado. En las filiaciones a pensiones, la cobertura varía entre el $39,8 \%$ y el $66 \%$. El último grupo, de países que presentan una menor cobertura, tanto en los niveles de salud, como en pensiones, son: Bolivia (Estado Plurinacional de), Guatemala, Honduras, Nicaragua y el Paraguay, con niveles en salud comprendidos entre el $36,3 \%$ y el 45,6 $\%$, y en pensiones entre el $34 \%$ y el $39,6 \%$.

Los sistemas de salud con la afiliación más igualitaria son, Uruguay seguido de Costa Rica, pero la afiliación al sistema de pensiones más igualitaria de los 18 países, lo tiene Costa Rica. Así, las afiliaciones a sistemas de pensiones y de salud en América Latina varían de $46,3 \%$ y $54,4 \%$ respectivamente, en 2002 , para $55,7 \%$ y $67,6 \%$, en 2013, con predominancia de afiliación de las mujeres.

En el ámbito de la asistencia social, la centralidad de políticas de combate a la pobreza generó una masificación y multiplicación de programas sociales focalizados (CEPAL, 2010). Sus características hacen parte de un contexto marcado por el énfasis, desde el período de 1980 y 1990, en los criterios de efectividad y eficiencia en la gestión de políticas y programas sociales focalizados, por el llamado a la participación de los individuos, de las familias, de las entidades sociales, en el fortalecimiento del "capital social" y de las habilidades emprendedoras. 


\section{Protección social latinoamericana: concepciones $y$ tendencias predominantes}

Sin embargo, a partir del final de los años 1990, los PTCs, gañan evidencia en el contexto da protección social, en la tentativa de dar una nueva cara al desarrollo. Su expansión se apoya en la concepción de desarrollo humano, difundida a partir del 1990, por el Programa de las Naciones Unidades para el Desarrollo (PNUD), como un proceso que debe ofrecer mayores oportunidades a las personas. Esa concepción orientó la formulación de programas y proyectos en el ámbito de las políticas de enfrentamiento a la pobreza. A partir de 2000, el Banco Mundial hace difusión de la concepción de protección social, definida como intervenciones públicas direccionadas a "asistir las personas, hogares y comunidades a mejorar su manejo del riesgo", así como "proporcionar apoyo a quienes se encuentran en la extrema pobreza" (HOLZMANN; JØRGENSEN, 2000, p. 3). Tal concepción "constituyó la principal rémora en términos conceptuales del reduccionismo en política social" (SOJO, 2017, p. 22), estructurada en la combinación público-privada, donde la responsabilidad del Estado en relación a protección social es mínima; al individuo, cabe el aseguramiento contra los riesgos en el mercado; la provisión de servicios para los pobres por medio de las redes de protección, cuya concepción fue también difundida por el Banco Interamericano de Desarrollo (BID), en 2000. La recomendación es que las acciones deben "enfocar el aumento de la base de activos de los pobres - en términos de capital humano e físico - incorporando incentivos de inversión en las transferencias de renda" (BID, 2000, p.151). Resalta, todavía, la importancia de la focalización y su eficiencia en "minimizar el desperdicio e evitar transferencias a los que no necesitan de apoyo público".

El consenso que orienta las referidas concepciones está en la comprensión de pobreza como "privación de capacidades básicas", o sea, la ausencia de un coyunto de bienes que una persona puede dominar para la expansión de su libertad en favor de la responsabilidad individual. Al Estado, como sugiere Amartya Sen (2000, p. 322), cabe "criar más oportunidades de elección y decisiones sustantivas para las personas". Sin embargo, para Sen (2000a) los esfuerzos deben ser conjugados a partir de un enfoque pluralista, o sea, del equilibrio entre el papel del Estado con el uso de los mercados (p. 159). De esa forma, 
la visión de desarrollo centrado en la producción de bienes da lugar a la visión centrada en la ampliación de las capacidades de las personas, tornándolas principales responsables por su desarrollo.

Se puede verificar en las proposiciones de los distintos organismos que las mismas, están dirigidas para desarrollar capacidades de las personas pobres. El punto de partida de Sen, como analiza Maranhão (2012), localizase en la renovación de las promesas liberales, al reconocer que solamente la economía del libre mercado puede ofrecer las oportunidades sociales capaces de permitir la construcción del desarrollo como libertad. Tal concepción transfiere el foco analítico centrado en la renta para otro foco centrado en las oportunidades sociales, con el objetivo de desarrollar las capacidades humanas de los agentes emprendedores individuales.

Al distinguir los términos, pobreza como falta de capacidad y pobreza como falta de ingreso, Sen (2000a) reconoce la inevitable relación entre ambos, considerando que el ingreso constituye un importante medio para alcanzar la capacidad. Mientras tanto, argumenta en favor del enfoque de la pobreza a partir de la privación de capacidades, confiando en que el "aumento de las capacidades de una persona para vivir, normalmente, tendería a aumentar su capacidad para ser más productiva y a percibir una renta más alta (p. 117). Por eso reitera sobre la necesidad de comprender "la pobreza y la privación desde el punto de vista de la vida que puede llevar realmente el individuo, y de las libertades que tiene en la realidad" (p. 119). Es por medio del mecanismo de mercado, considerado por Sen (2000a) como una institución básica, que los individuos pueden interrelacionarse y realizar actividades mutuamente ventajosas y, así, expandir sus libertades (p. 178).

Los organismos internacionales anteriormente destacados, y sus recomendaciones, viabilizan en 2000, una nueva versión del Consenso de Washington, que se materializa por medio de medidas para alcanzar determinadas metas y Objetivos de Desarrollo del Milenio (ODM). Desde entonces, la pobreza pasa a ser considerada el principal problema, y foco, de las políticas sociales. Estas abandonan la perspectiva de la universalización del acceso a servicios básicos para incorporar, con centralidad, la reducción o enfrentamiento a la pobreza y, con ella, nuevos instrumentos y concepciones, tales como: la igualdad de oportunidades, por medio de la focalización y selección de los beneficiarios; 
de la transferencia monetaria o en efectivo, pero, condicionada a la contraprestación y activación de las/los beneficiarias/os.

En el referido contexto, ya en la mitad de la última década, las referidas instituciones alientan con la posibilidad de "una nueva política social para la América Latina" (BID, 2006), rescatando conceptos fundamentales para la ciudadanía social, sin embargo, dando a ellos nuevos significados. Con el argumento de la escasez de recursos, surgen propuestas como el universalismo básico (BID) y del Piso de Protección Social (OIT, 2011), pero desfiguradas de la verdadera concepción universal del principio de la universalidad, de connotación eminentemente pública, ciudadana e igualitaria (PEREIRA; STEIN, 2010, p. 107), como puede ser observado: el universalismo básico, a pesar de definido como "una cobertura universal de prestaciones y riesgos", pretendiese que lo sea a partir de "un coyunto limitado de prestaciones básicas, que incluye las prestaciones esenciales del derecho universal, que variará de acuerdo con las posibilidades y definiciones propias de cada país". (FILGUEIRA et al., 2006, p. 21).

De la misma forma, la concepción sobre el piso de protección social, desarrollado por la Organización Internacional del Trabajo (OIT) y la Organización Mundial de la Salud (OMS) como "un coyunto integrado de políticas sociales diseñado para garantizar a toda persona la seguridad de los ingresos y el acceso a los servicios sociales esenciales, prestando especial atención a los grupos vulnerables y protegiendo y empoderando a las personas a lo largo del ciclo de vida" (OIT, 2011, p. 9). Sin embargo, las propuestas recientes y las reducidas experiencias en la perspectiva de la integralidad se muestran todavía tímidas y limitadas para impactaren sobre el tamaño de la miseria y de la desigualdad que asola la región.

El PNUD en su Informe Anual de 2013, presenta la concepción de Estado desarrollista, en la cual el "debe estar centrado en los ciudadanos, promoviendo oportunidades y brindando protección contra riesgos inconvenientes" (2013, p. 68). Sugiere, por lo tanto, que relacionemos con el proceso de crecimiento ocurrido en la última década. $\mathrm{O}$ sea, la mejora de diversos indicadores sociales, principalmente, los de la pobreza con base en la renta, tras las privaciones sociales visibles con la "globalización de la pobreza" y mercantilización de la protección social 
en América Latina, son presentados como el retorno a la era desarrollista y, supuestamente, post-neoliberal.

Desde el compromiso internacional con las metas establecidas por los ODM, en 2000, se verifica la expansión y centralidad de los Programas de transferencias monetarias asistenciales (condicionadas o no) en el contexto de las políticas sociales direccionadas para la pobreza. A partir de 2015, 17 nuevos objetivos con 169 metas pasan a componer la Agenda 2030 para el Desarrollo Sostenible que abarcan las esferas económica, social y ambiental.

La implementación de las Agendas constituyo uno de los factores de la ampliación del gasto social público y de su mayor participación en el PIB. De acuerdo con Tromben (2016, p. 9), el gasto social viene creciendo "desde $11,3 \%$ del producto interno bruto en promedio simple en 1990 hasta 14,7\% del PIB en 2014". Afirma, todavía, que los mayores aumentos ocurrirán a partir de la segunda mitad de los ańos 2000, siendo su mayor gasto social, desde entonces, correspondiente al de seguridad social que pasó de 3,0\% del PIB en 1990 al 5,6\% del PIB en 2014.

La evolución del gasto por funciones sociales a nivel del sector público, analizados por CEPAL (2017), destaca que la asignación de recursos prioritarios, fueron destinadas a la protección social, educación y salud como promedio de los países considerados, al 5,0\%, el 4,6\% y el 3,4\% del PIB, respectivamente, en 2015. El análisis del gasto social realizado no periodo de 2000 a 2015, así como, el gasto presupuestado por funciones del gobierno central correspondiente a los años fiscales 2015, 2016 y 2017, evidenció que los países de la región "se han mostrado cautelosos al elaborar los presupuestos de 2016 y 2017, en el sentido de que han presupuestado gastos sociales - así como gastos totales - menores que los de 2015” (p. 17).

Frente a esta "cautela" de los gobiernos, y de la reducción creciente desde 2013, de la inversión, incluso, en políticas focalizadas, la recomendación creciente de parte de los organismos internacionales es la combinación de transferencia monetaria con políticas y programas de inclusión laboral y productiva, por medio de, entre otras medidas, la capacitación y formación técnica y profesional, en conformidad con la Agenda 2030 para el Desarrollo Sostenible, meta 8.3 que recomienda 
"Promover políticas orientadas al desarrollo que apoyen las actividades productivas, la creación de puestos de trabajo decentes, el emprendimiento, la creatividad y la innovación, y fomentar la formalización y el crecimiento de las microempresas y las pequeñas y medianas empresas, incluso mediante el acceso a servicios financieros" (CEPAL, 2016, p. 115). Esta Agenda hace parte de las políticas que, en Europa, son denominadas de "Políticas de Activación".

Teniendo en cuenta la relevancia asumida por los PTCs, en la reducción de indicadores de pobreza, pasamos a una breve configuración de los mismos en América Latina.

\section{Los PTCs en América Latina}

Transcurridos veinte años de la implementación de los primeros PTCs en América Latina, mucho ya se ha producido sobre su expansión como estrategia de gestión de la pobreza no solo en América Latina, pero también, en varias partes del mundo. Para conocer las diversas características de los referidos PTCs recorremos, entre otras, a siguientes fuentes: Cechini y Atuesta (2017); Cepal (2017, 2013); Cecchini y Martinez (2011); Cecchini y Madariaga (2011); Rodríguez Enríquez (2011); Fiszbein e Schady (2009).

Las primeras experiencias con programas de transferencia monetaria condicionada tuvieron inicio en 1995, en algunos municipios de Brasil, y también en la experiencia piloto realizada en Campeche, Mexico. Pero fue en 1997, en México, que se inició la primera experiencia de amplitud nacional. A partir de entonces otros países pasaron a desarrollarlo, ganando destaque a partir del año 2000, con la Declaración de los Objetivos del Milenio. Actualmente, la región cuenta con 30 PTCs activos en 20 países. (CECCHINI; ATUESTA, 2017).

A pesar de la heterogeneidad de las experiencias en la región, hay rasgos comunes entre ellos, como la transferencia monetaria a familias en situación de pobreza o extrema pobreza, con uno o más hijos menores de edad, mediante el cumplimiento de condicionalidades, asocia-

5 Tratase de políticas e instrumentos con el objetivo de integrar en el mercado de trabajo a las personas desempleadas perceptoras de prestaciones económicas y a mejorar sus niveles de integración social y económica. (SIIS, 2012). 
das directamente a dos objetivos: uno, de corto plazo, que consiste en aliviar la situación de pobreza por renda, con el aumento de recurso para satisfacer necesidades básicas; y, otro, de largo plazo, visando contribuir para el fortalecimiento de las capacidades de niñas, niños y adolescentes, con el objetivo de evitar la reproducción intergeneracional de la pobreza. Un aspecto de grande relevancia en el debate de los PTCs está relacionado al cumplimiento de condicionalidades, así como el vínculo entre la familia y el Programa. En general, requieren el compromiso con la asistencia a escuela, de niñas y niños en edad escolar, y al control de salud, de aquellos en edad preescolar.

Partiendo del enfoque asumido por cada PTC, cuanto a los objetivos de corto y de largo plazo, sus prestaciones, y la lógica con que operan las condicionalidades, diversos autores (CECCHINI, MARTINEZ, 2011; CECCHINI, MADARIAGA, 2011) apuntan para la configuración de una tipología de PTC en América Latina. Cuanto a los objetivos, hay PTCs que enfatizan los de corto plazo y priorizan la provisión de renta para asegurar la satisfacción de niveles mínimos de consumo, en cuanto otros enfatizan los objetivos de largo plazo y, así, priorizan la generación y acumulación de capital humano. Otra distinción se refere al papel de las transferencias monetarias en la lógica de funcionamiento del Programa: aumentar la renta actual de los beneficiarios, o actuar como incentivo. Dependiendo de la priorización adoptada, los PTCs presentan distinción cuanto al tipo de transferencia, al cálculo de su valor y sus límites.

Otro aspecto se refiere a los tipos o formas asumidas por la condicionalidad, o sea: la justificativa utilizada para su inclusión o, las modalidades (control, monitoreo y sanciones) y quien asume responsabilidad en su cumplimiento. Autores como Cecchini e Madariaga (2011), Cecchini e Martinez (2011), distinguen ${ }^{6}$ tres tipos de condicionalidades que generan los diferentes tipos de PTCs, para tanto, toman como referencia las experiencias de México, Brasil y Chile, para comprender el lugar que los mismos ocupan en el contexto de la protección social. Fiszbein y Schady (2009), por su vez, identifican la varia-

6 Cecchini y Madariaga (2011) denominan las condicionalidades, como fuerte, moderada y leve, en cuanto Cecchini y Martinez (2011) denominan, respectivamente, de fuerte, leve y sistemas o redes de coordinación programática con condicionalidades. 
ción de los PTCs a partir de los tipos de control y sanciones relacionadas al incumplimiento de las condicionalidades (control ligero, esporádico y frecuente; sanciones suaves, leves y fuertes).

Entre los trazos comunes a los PTCs, además del cumplimiento de condicionalidades vinculadas a salud, educación y asistencia, pasa a ampliar las transferencias no monetarias vinculadas a inserción laboral y generación de renta; estímulo a capacidades básicas y desarrollo humano de los usuarios; orientación familiar y trabajo psicosocial; infraestructura (programa de mejoría en comunidades y barrios).

La cobertura de los PTCs en la región pasó de menos de un millón en 1996 a los 131,8 millones en 2015, representando al 20,9\% de la población regional. De la misma forma, el número de hogares destinatarios de los PTC pasó de menos de 300.000 hogares en 1997 a los 29,8 millones en 2015, correspondiente al 17,5\% del total de hogares de la región (CECCHINI; ATUESTA, 2017). Sin embargo, desde 2014, observase la tendencia a reducción de esta cobertura, en que pese las estimativas de CEPAL de aumento de la pobreza en 2016.

El programa con mayor cobertura absoluta de hogares en la región es el Bolsa Familia de Brasil, que pasó de 3,6 millones de hogares en 2003 a 14,1 millones de hogares en 2013, bajando a 13.284.029 de hogares en junio de 2017. En seguida, la mayor cobertura es del Programa Mexicano, que tuvo inicio con el Progresa (1997-2001) con una cobertura de 1,6 millones de hogares, sustituido por el Oportunidades (2001-2014), que se siguió del Prospera (desde 2014) con 6,1 millones de hogares en 2016. El siguiente es el Programa Colombiano, Más Familias en Acción, que en 2014 alcanzó 2,7 millones de familias y en 2016 hubo una reducción para 2,5 millones de familias; seguido del Programa Argentino Asignación Universal por Hijo para Protección Social, que en 2016 alcanzó a 2,0 millones de hogares. Verificase una ampliación significativa en la cobertura del Programa Juntos en Perú, pasando de 22.500 hogares cubiertos en 2005 a 769.000 en 2015, pero, reduciendo a 668.000 en 2016. (CECCHINI; ATUESTA, 2017; BRASIL, MDS, 2017).

Sin embargo, cuando se observa la cobertura en términos proporcionales a la población nacional, véase que los Programas Bono Juancito Pinto (BJP) y Bono Juana Azurduy (BJA) del Estado Plurinacional 
de Bolivia alcanzaron una cobertura del 37\% de la población en 2005 pasando al 61\% en 2015. También el Programa Progresando con Solidaridad (hasta 2012, Solidaridad) de la República Dominicana, aumentó su cobertura, pasando del 8,7\% de su población en 2005 al $31,3 \%$ en 2015. La cobertura de la población en América Latina y el Caribe, por parte de los PTC, ha tenido un aumento de 3,6\% en 2000 al $20,9 \%$ en 2015.

Dimensionando sobre el nivel de inversión regional en los PTCs, presentado por Cecchini y Atuesta (2017) verificase que en 2015 hubo la inversión del 0,33\% del PIB. Los países que más invirtieron fueron: Argentina (0,59\% del PIB), Brasil (0,50\% del PIB), República Dominicana $(0,43 \%$ del PIB) y Uruguay $(0,39 \%$ del PIB), en cuanto el Programa de Ecuador, el Bono de Desarrollo Humano de Ecuador presenta el porcentual de mayor inversión, el 0,66\% del PIB, pero llama a la atención el hecho de que el País, además de la transferencia monetaria condicionada para familias con nińos (alcanza al 0,26\% del PIB), incluye también la pensión para adultos mayores y la pensión para personas con discapacidad. Autores destacan el crecimiento de la inversión en porcentaje del PIB en toda la región en las dos últimas décadas, como porcentaje del gasto público social, observándose que la inversión regional en PTC aumentó de 0,04\% en 1997 a 3,1\% en 2015.

Las características de los PTCs en América Latina dejan ver la lógica común de la focalización en la pobreza extrema y la exigencia de contrapartidas, que vinculase a meritocracia y a ideología del esfuerzo. Como afirma Otero (2014, p. 52) esa ideología "supone el abandono de la seguridad proporcionada por el Estado en favor de la independencia individual, del riesgo y de las recompensas para quien, superados los obstáculos, alcancen el éxito".

El uso de la focalización, condicionada por el principio de la menor elegibilidad, acarrea diversas implicaciones, no solo desde el punto de vista operacional, pero también, político, como resaltan Pereira y Stein (2010). La posibilidad de reducción de eficacia de las políticas contra la pobreza, del punto de vista operacional: a) correcta identificación de las necesidades de cada grupo y la jerarquización de las situaciones; b) elevación de los costos administrativos; c) posibilidad de generación de efectos como el asistencialismo. 
Del punto de vista político, presenta vicios que constriñen, como la comprobación de la pobreza (testes de medios); la desconfianza cuando el ciudadano pobre recorre a protección del Estado, como se fuera un fraudador (fraudemania); condicionalidades o contrapartidas, que transforman el derecho en concesión; el estigma, que "no liberta el ciudadano de derecho de la situación de privación", como afirma Pereira (2009, p. 7). En concordancia con Pereira (2009) y Boschetti (2016), las políticas focalizadas constituyen una "trampa de la pobreza", pues, para que tengan acceso a la política, el ciudadano pobre hay que mantenerse en la situación de pobreza absoluta.

\section{Asistencia Social, expropiación y activación para el trabajo}

La centralidad, en las dos últimas décadas, de los programas de transferencias monetarias condicionadas y focalizadas, de la política de asistencia y de enfrentamiento a la pobreza, pone en evidencia la tensa relación entre asistencia social y trabajo. La protección al largo de la historia demostró dos formas distintas de acción, una, contributiva, dirigida a los aptos para el trabajo y, otra, asistencial, para los pobres incapaces. Sin embargo, la asistencia social en los tiempos actuales, aparentemente, parece romper con esta separación considerando la incorporación de trabajadores en condiciones de trabajar. Mientras tanto la expansión de la asistencia social, en especial, los PTC, ocurre en contexto de restructuración del Estado social, de expropiación de los trabajadores y precarización del trabajo, de mercantilización de los sistemas públicos y supresión de derechos, de hegemonía del capital financiero. Así, programas de esta naturaleza, pasan a función capitalista de reproducción de la superpoblación relativa y al equilibrio la relación producción/consumo de modo rentable. (BOSCHETTI, 2016, 166; MOTA; TAVARES, 2016, p. 233).

La "relación de atracción y rechazo entre trabajo y asistencia social” (BOSCHETTI, 2016, p. 167), aparentemente resuelta con la incorporación de trabajadores en condiciones de trabajar en programas sociales, es deconstruida, cuando el Estado reclama al individuo salir de su "pasividad", por medio de la sustitución del cumplimiento de lo que siempre se denominó de condicionalidades, para su "participación” en la contrapartida. Prevalece la identificación con la ética del workfare, de la inserción en el mercado de trabajo como fuente de 
bienestar, como capaz de liberar el pobre de la pobreza y así, no crear "dependencia" de los individuos en relación con las prestaciones sociales. O sea, de creedor de derechos sociales, pasan a ser responsable de si mismo y deudor de la asistencia, de titular de derecho a un simple beneficiario. La moralización y individualización como principio de la activación, objetivan cambiar conductas y actitudes individuales, donde la cultura emprendedora es apuntada como la "puerta de salida" y alternativa para la "autonomía" económica.

Artigo submetido em 31 de julho de 2017 e aceito para publicaçáo em 15 de agosto de 2017

\section{Referencias}

BID. Protección social para la equidad y el crecimiento. BID: Washington, D.C. 2000.

- Realidades e perspectivas. Pulso social da América Latina e Caribe 2016. BID: Washington-DC, 2016.

BOSCHETTI, I. S. Assistência social e trabalho no capitalismo. São Paulo: Cortez, 2016.

BRASIL. MDS. SAGI. Relatórios de Informaçôes Sociais. RI Bolsa Família e Cadastro Único. Disponível em: <https://aplicacoes.mds.gov.br/sagi/ portal/>. 2017 .

CECCHINI, S.; ATUESTA, B. Programas de transferencias condicionadas en América Latina y el Caribe. Tendencias de cobertura e inversión. Serie Políticas Sociales, n. 224. CEPAL, 2017

CECCHINI, S.; MARTÍNEZ, R. Protección social inclusiva en América Latina. Una mirada integral, un enfoque de derechos. Naciones Unidas, Santiago de Chile, 2011.

CECCHINI, S; MADARIAGA, A. Balance de la experiencia reciente en América Latina y el Caribe. Cuadernos de la CEPAL 95. 2011.

CEPAL. Comisión Económica para América Latina y el Caribe. La hora de la igualdad: brechas por cerrar, caminos por abrir. Naciones Unidas, Santiago. Disponible en: <http://www.eclac.cl>. 2010.

. Panorama social de América Latina 2015. Naciones Unidas.

Disponible en: <www.cepal.org>. 2016. 
. Panorama Social de América Latina 2016. Naciones Unidas. Disponible en: <www.cepal.org>. 2017.

. Panorama Social de América Latina 2012. Disponible en: http:// www.eclac.cl. 2013

FILGUEIRAS, F. et al. Universalismo básico: una alternativa posible y necesaria para mejorar las condiciones de vida. En: Universalismo básico. Una nueva política para América Latina. MOLINA, C. G. (Ed.). Washington, BID: Planeta, 2006.

FILGUEIRAS, F. Los regímenes de bienestar en el ocaso de la modernización conservadora: posibilidades y límites de la ciudadanía social en América Latina. Revista Uruguaya de Ciencia Política, v. 22, n. 2, p. 17-46, 2013.

FISZBEIN, A.; SCHADY, N. Panorama General. Transferencias monetarias condicionadas: reduciendo la pobreza actual y futura. Banco Internacional de Reconstrucción y Fomento. Banco Mundial. Waschington, DC. 2009.

FRANCO, R. Los paradigmas de la política social en América Latina. Revista de la CEPAL, n. 58, LC/G.1996, Naciones Unidas, Santiago de Chile, p. 0922. Disponible en: <http://www.cepal.org/es/publicaciones/37982-revista-lacepal-no58>. Acceso en: 20/06/2017.

GRANEMANN, S. Políticas sociais e financeirização dos direitos do trabalho. Em Pauta. n. 20, 2007.

HOLZMANN, R.; JØRGENSEN, S. Manejo social del riesgo: un nuevo marco conceptual para la protección social y más allá. 2000. Disponible en: <http://wbln0018.worldbank.org/HDNet/HDdocs. nsf/655538a343139acab>. Acceso en: 20/05/2017.

MARANHÃO, C. H. Desenvolvimento social como liberdade de mercado: Amartya Sen e a renovação das promessas liberais. En: MOTA, A. E. (Org.). Desenvolvimento e construção de hegemonia: crescimento econômico e reprodução da desigualdade. São Paulo: Cortez, p. 78-104, 2012.

MOREnO, L. A. A década da América Latina e do Caribe: uma oportunidade real. Banco Interamericano de Desenvolvimento (BID), Washington, 2011.

MOTA, A. E.; TAVARES, M. A. Trabalho e expropriações contemporâneas. In: MOTA, A. E; AMARAL, A. (Orgs.). Cenários, contradiçóes e pelejas do Serviço Social brasileiro. São Paulo: Cortez, 2016, p. 229-251.

OIT. Oficina Internacional del Trabajo. Piso de Protección Social para una globalización equitativa e inclusiva. Informe del Grupo consultivo sobre el Piso de Protección Social. Ginebra, 2011. 
OTERO, José Moya. La ideología del esfuerzo. Madrid: La Catarata, 2014. PEREIRA, P. A. P.; STEIN, R. H. Política Social: universalidade versus focalização. Um olhar sobre América Latina. En: BOSCHETTI, I et al.. (Org.). Capitalismo em crise, política social e direitos. São Paulo: Cortez, p. 106-130, 2010.

PEREIRA, P. A. P. A assistência social na perspectiva dos direitos. Crítica aos padrōes dominantes de proteção social aos pobres no Brasil. Brasília: Thesaurus, 1996.

- Proteção social contemporânea: cui prodest? Serviço Social e Sociedade, n. 116, out.-dez./2013,

. Políticas Sociais: focalização X universalização. Politizando. Ano 1, 1. ed., 2009. Disponible en: <https://www.neppos.com/politizando>. Aceso en: 07/2017.

PNUD. Informe Desarrollo Humano. New York, EUA: Naciones Unidas, 2000.

Informe sobre Desarrollo Humano 2013. El acenso del sur: progreso humano en un mundo diverso. New York, EUA: Naciones Unidas, 2013.

RODRIGUEZ ENRIQUEZ, C. Programas de transferências condicionadas de ingreso e igualdad de género ¿Por donde anda América Latina? CEPAL, Serie Mujer y desarrollo, n. 109, 2011.

SEN, Amartya. Desenvolvimento como liberdade. Tradução de Laura Teixeira Motta. São Paulo: Companhia das Letras, 2000.

Desarrollo y libertad. Trad. Esther Rabasco y Luis Toharia. Barcelona: Planeta, 2000a.

SIIS. Centro de Documentación y Estudios. Activación y derecho a la inclusión en el marco de las políticas de empleo y de garantía de ingresos en la CAPV. Fundación Eguía-Careaga. Bilbao, Espanha, 2012.

SOJO, Ana. Protección social en América Latina: la desigualdad en el banquillo, Libros de la CEPAL, n. 143 (LC/PUB.2017/7-P), 2017.

TROMBEN, Varinia. Gasto social y ciclo económico en América Latina y el Caribe. CEPAL. Serie Políticas Sociales, n. 219, 2016. 